\title{
Template Epitaxial Growth of Thermoelectric Bi/BiSb Superlattice Nanowires by Charge-Controlled Pulse Electrodeposition
}

\author{
Xincun Dou, ${ }^{a}$ Guanghai Li ${ }^{\mathrm{a}, \mathrm{z}}$ Hechang Lei, ${ }^{\mathrm{a}}$ Xiaohu Huang, ${ }^{\mathrm{a}}$ Liang $\mathrm{Li},{ }^{\mathrm{a}}$ and \\ Ian W. Boyd ${ }^{\mathrm{b}}$ \\ ${ }^{a}$ Key Laboratory of Materials Physics and Anhui Key Laboratory of Nanomaterials and Nanotechnology, \\ Institute of Solid State Physics, Chinese Academy of Sciences, Hefei 230031, People's Republic of \\ China \\ ${ }^{b}$ Department of Electronic and Electrical Engineering, University College London, London WC1E 7JE, \\ United Kingdom
}

\begin{abstract}
$\mathrm{Bi} / \mathrm{BiSb}$ superlattice nanowires (SLNWs) with a controllable and very small bilayer thickness and a sharp segment interface were grown by adopting a charge-controlled pulse electrodeposition. The deposition parameters were optimized to ensure an epitaxial growth of the SLNWs with a preferential orientation. The segment length and bilayer thickness of the SLNWs can be controlled simply by changing the modulating time, and the consistency of the segment length can be well maintained by our approach. The Bravais law in the electrodeposited nanowires is verified by the SLNW structure. The current-voltage measurement shows that the SLNWs have good electrical conductance, particularly those with a smaller bilayer thickness. The Bi/BiSb SLNWs might have excellent thermoelectric performances.

(c) 2009 The Electrochemical Society. [DOI: 10.1149/1.3156639] All rights reserved.
\end{abstract}

Manuscript submitted March 16, 2009; revised manuscript received May 19, 2009. Published July 6, 2009.

Nanostructured thermoelectric materials can have dramatically higher efficiencies than their bulk counterparts. ${ }^{1-9}$ The effectiveness of a thermoelectric material could be linked in an approximate way to the dimensionless thermoelectric figure of merit, $Z T=S^{2} \sigma T / \kappa$, where $S, \sigma, T$, and $\kappa$ are, respectively, the Seebeck coefficient, the electrical conductivity, the temperature, and the thermal conductivity. ${ }^{1}$ Compared to simple quantum wires, quantum dots, quantum wells, and two-dimensional superlattices, the quantum dot superlattices and superlattice nanowires (SLNWs) exhibit even greater advantages in the enhancement of the figure of merit $(Z T) .^{3}$ Because the heterogeneous interfaces between the nanodots can block the phonon conduction along the wire axis and thus reduce the lattice thermal conductivity, while the electrical conduction can be sustained and may benefit from the unusual electronic band structures, the SLNWs are especially attractive for thermoelectric applications. ${ }^{10}$ Recent studies further proved that the increased phonon scattering by grain boundaries would increase the $Z T$ value. ${ }^{2,8,9,11}$ The electron and phonon properties of SLNWs can be manipulated by many parameters, such as segment length, wire diameter, crystal orientation, bilayer thickness, and so on.

Different methods have been adopted to fabricate SLNWs, such as $\mathrm{Si} / \mathrm{SiGe}^{12}$ and $\mathrm{GaAs} / \mathrm{GaP}^{13}$ by chemical vapor deposition and laser-assisted catalytic growth, and InP/InAs ${ }^{14}$ by chemical beam epitaxy. Electrodeposition is a promising alternative technique for the fabrication of SLNWs ${ }^{15}$ and several kinds of SLNWs have been fabricated in a single bath based on electrochemical growth in porous films. ${ }^{16-25}$ However, all these SLNWs are synthesized by modulating direct electrodeposition, the crystallinity is relatively not very high, and the interfaces between segments are not distinct. The pulsed electrodeposition, with various processing parameters and higher instantaneous current density, exhibits marked advantages over dc electrodeposition in controlling the deposited grain size, surface morphology, and preferred orientation, and proved to be a viable technological tool in materials engineering. ${ }^{26,27}$

$\mathrm{Bi}$ is especially favorable for studying low dimensional systems and for low temperature thermoelectric applications due to its small electron effective mass and highly anisotropic Fermi surface. ${ }^{28} \mathrm{Cal}-$ culations have proved that an unprecedented enhancement of the ZT value could be obtained in the $\mathrm{Bi}_{1-x} \mathrm{Sb}_{x}$ alloy nanowire system with the variation in $x$ and the diameter of nanowires compared to pure $\mathrm{Bi}$ nanowires. ${ }^{29}$ Some promising work has been done on the $\mathrm{Bi}_{1-x} \mathrm{Sb}_{x}$

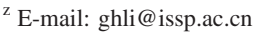

alloy $^{30-33}$ and other Bi-based ${ }^{34,35}$ nanowire systems. As a result, the design and fabrication of SLNWs composed of $\mathrm{Bi}_{1-x} \mathrm{Sb}_{x}$ segment nanowires is especially desirable for thermoelectric applications. The ZT of SLNWs generally increases with a decrease in both the wire diameter and the segment length before the alloy limit is reached, ${ }^{10}$ and thus the growth of SLNWs with small bilayer thickness and sharp segment interface is essential in achieving a better thermoelectric performance. However, the previous endeavor has been met with a great challenge. ${ }^{24,25}$ Here we report the epitaxial growth of Bi/BiSb SLNWs with a controllable and very small bilayer thickness and sharp segment interface by adopting a chargecontrolled pulse electrodeposition.

\section{Experimental}

Chemicals. - The anodic alumina membrane (AAM) used was prepared using the same procedure as in our previous report. ${ }^{30}$ The pore size of the AAM was about $60 \mathrm{~nm}$. The electrolyte for the deposition of $\mathrm{Bi} / \mathrm{Bi}_{1-x} \mathrm{Sb}_{x}$ SLNWs contained a mixture of $0.04 \mathrm{M}$ $\mathrm{BiCl}_{3}, 0.08 \mathrm{M} \mathrm{SbCl}_{3}, 0.27 \mathrm{M}$ tartaric acid, $0.24 \mathrm{M}$ citric acid, $0.1 \mathrm{M}$ glycerol, $1.2 \mathrm{M} \mathrm{NaCl}$, and $1.0 \mathrm{M} \mathrm{HCl}$. The $\mathrm{pH}$ value of the electrolyte was adjusted to about 0.82 by adding appropriate amounts of 5 $\mathrm{mol} / \mathrm{L}$ aqueous ammonia. The deposition temperature was maintained at about $36^{\circ} \mathrm{C}$ with a water bath.

Instruments and measurements.- Power X-ray diffraction (XRD; Philips PW 1700x with $\mathrm{Cu} \mathrm{K \alpha}$ radiation), field-emissionscanning electron microscopy (FESEM; FEI Sirion-200), transmission electron microscopy (TEM), high resolution transmission electron microscopy (HRTEM; JEOL-2010), and selected area electron diffraction (SAED) were used to study the crystalline structure and morphology of the SLNW array. The chemical composition was determined by energy-dispersive spectrometry (EDS). For XRD measurements, the overfilled nanowires on the surface of the AAM were mechanically polished away. For scanning electron microscopy (SEM) observations, the AAM was partly dissolved with a $0.5 \mathrm{M}$ $\mathrm{NaOH}$ solution and then carefully rinsed with deionized water several times. For TEM observations, the AAM was completely dissolved with a $1 \mathrm{M} \mathrm{NaOH}$ solution and then rinsed with absolute ethanol.

The annealing treatment was performed in high vacuum at $120^{\circ} \mathrm{C}$ for $10 \mathrm{~h}$. The current-voltage $(I-V)$ response of the SLNW arrays was carried out by a Keithley 4200 Semiconductor Characterization System, and the $I-V$ response of individual SLNW was performed by Omicron low temperature scanning tunneling microscope. 

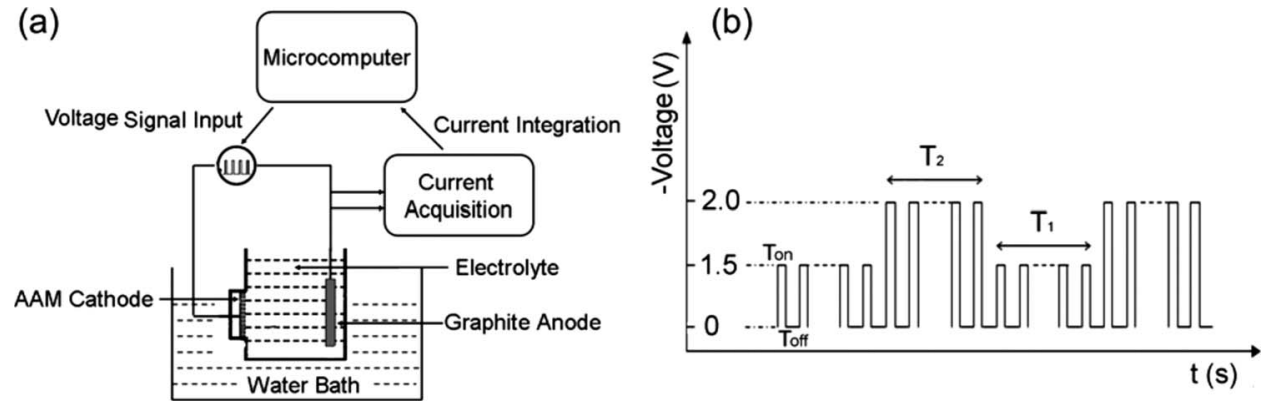

Figure 1. (a) Schematic illustration of experimental setup. (b) Schematic diagram of the voltage pulses used for the deposition of $\mathrm{Bi} / \mathrm{Bi}_{0.5} \mathrm{Sb}_{0.5}$ SLNWs. $T_{1}$ and $T_{2}$ represent the modulating times for the growth of $\mathrm{Bi}$ and $\mathrm{Bi}_{1-x} \mathrm{Sb}_{x}$ alloys, respectively. $T_{\mathrm{on}}=8 \mathrm{~ms} ; T_{\mathrm{off}}=12 \mathrm{~ms}$.
Charge-controlled pulse electrodeposition.- Figure 1a schematically illustrates the experimental setup for the growth of $\mathrm{Bi} / \mathrm{Bi}_{1-x} \mathrm{Sb}_{x}$ SLNWs, which contains a personal computer (PC)-controlled pulse voltage signal input unit, a real-time current acquisition system (RTCAS), and a two-electrode plating cell in which the AAM sputtered with a layer of $\mathrm{Au}$ films (about $200 \mathrm{~nm}$ thick) serves as the cathode and a graphite plate serves as the counter electrode. The PCcontrolled pulse voltage signal input unit could provide the necessary pulse voltage waveform for electrodeposition. The twoelectrode arrangement with a pulse electrodeposition technique proved to be a simple and facile method to fabricate Bi-based nanowires, such as $\mathrm{Bi}, \mathrm{Sb}$, and BiSb alloy nanowire arrays. The twoelectrode arrangement with dc electrodeposition technique was also applied to effectively fabricate $\mathrm{Co}, \mathrm{Ni}$, and $\mathrm{Ag}$ nanowire arrays.

The current slightly increased during the growth of nanowires in the AAM pores due to the decreased distance between the front of the nanowire and the pore opening when the voltage controlling was adopted. $^{21}$ The thickness of the $\mathrm{Ni} / \mathrm{Cu}$ bilayers increased with the growing nanowires although the modulating time was kept constant. ${ }^{18}$ To ensure a uniform bilayer thickness along the whole SLNW, we used the electrical charge transferred during the pulsation voltage to control the segment length of each component. Each cathode voltage was set by a PC-controlled pulse voltage signal generator; the PC instantaneously integrated the current that the RTCAS acquired to get the electric charge passed during each modulating time and monitored the subsequent pulse voltage signal generator.

Figure $1 \mathrm{~b}$ shows the input pulse voltage signal for the deposition of Bi/BiSb SLNWs, in which a constant pulse time $T_{\text {on }}$ of $8 \mathrm{~ms}$ and a delayed time $T_{\text {off }}$ of $12 \mathrm{~ms}$ were used for the deposition of both pure $\mathrm{Bi}$ and BiSb alloys. $T_{1}$ and $T_{2}$ represent the modulating time of the first 10 cycles for the deposition of Bi and BiSb alloys at voltages of -1.5 and $-2.0 \mathrm{~V}$, respectively. The charge passed in each voltage of the 10th deposition cycle was set as a standard charge to control the following segment length. The length of $\mathrm{Bi}$ and $\mathrm{BiSb}$ segment nanowires $L_{1}$ and $L_{2}$ could be controlled by changing $T_{1}$ and $T_{2}$, upon which the bilayer thickness $\left(L=L_{1}+L_{2}\right)$ of the Bi/ BiSb SLNWs could be easily adjusted. During the pulse time $T_{\text {on }}$, metal species were reduced at the cathode, and no deposition proceeded in the course of the relaxation time $T_{\text {off. }}$ The pulse time in each pulse cycle was very short; only a small amount of metal ions was reduced at the reaction interface during one pulse (less than one atom layer.) The metal ion concentration changed slightly near the reaction interface per pulse. Moreover, the delayed time was sufficient for the concentration of metal ions to recover at the reaction interface. There was no evident concentration gradient during the deposition. Thus, the pulse time that controlled the transport of ions dominated the growth rate of the nanowires. The pulsed electrodeposition can improve the homogeneity of the deposition and can limit the hydrogen evolution. ${ }^{36}$ The surface lattice of the segment nanowires at the metal-solution interface could relax to a more stable state, and those atoms from the surface lattice having the lowest binding energies could be removed, which would otherwise lead to lattice imperfections. ${ }^{37}$ This atom-by-atom growth favors the perfect crystalline quality and preferentially oriented growth of the nanowires.

Deposition parameter optimization.- To optimize the parameters for the growth of the Bi/BiSb SLNWs, the deposition voltages ranged from -1.4 to $-2.1 \mathrm{~V}$, and $T_{\mathrm{on}}$ and $T_{\text {off }}$ from hundreds of microseconds to hundreds of milliseconds could be used to deposit the alloy nanowires; the voltages of -1.5 and $-2.0 \mathrm{~V}$, and $T_{\text {on }}$ of 8 $\mathrm{ms}$ and $T_{\text {off }}$ of $12 \mathrm{~ms}$ were optimal parameters to deposit pure $\mathrm{Bi}$ and $\mathrm{Bi}_{0.5} \mathrm{Sb}_{0.5}$ alloy nanowires, respectively. Figure 2a shows the XRD pattern of the Bi nanowire array together with the standard diffraction peaks of Bi (JCPDS Card no. 85-1331), in which all diffraction peaks can be indexed to the rhombohedral space group $R \overline{3} \mathrm{~m}$ (to which element Bi belongs). The sharp peak situated at $39.79^{\circ}$ indicates that the obtained Bi nanowire array was highly and preferentially oriented along the [110] direction. The EDS profile and the corresponding FESEM image shown in Fig. $2 b$ prove that the obtained nanowire is pure $\mathrm{Bi}$ and has a diameter of about $60 \mathrm{~nm}$. Figure $2 \mathrm{c}$ shows the XRD pattern of the $\mathrm{Bi}_{0.5} \mathrm{Sb}_{0.5}$ alloy nanowire array together with the standard diffraction peaks of BiSb (JCPDS Card no. 35-0517), in which all diffraction peaks also can be indexed to the rhombohedral space group $R \overline{3} m$ (to which the $\mathrm{Bi}_{1-x} \mathrm{Sb}_{x}$ alloy belongs). The sharp peak situated at $41.31^{\circ}$, which is between the peaks for elements $\mathrm{Bi}$ and $\mathrm{Sb}$, is firm evidence of the formation of the $\mathrm{Bi}_{1-x} \mathrm{Sb}_{x}$ alloy. The EDS spectrum is shown in Fig. 2d, and
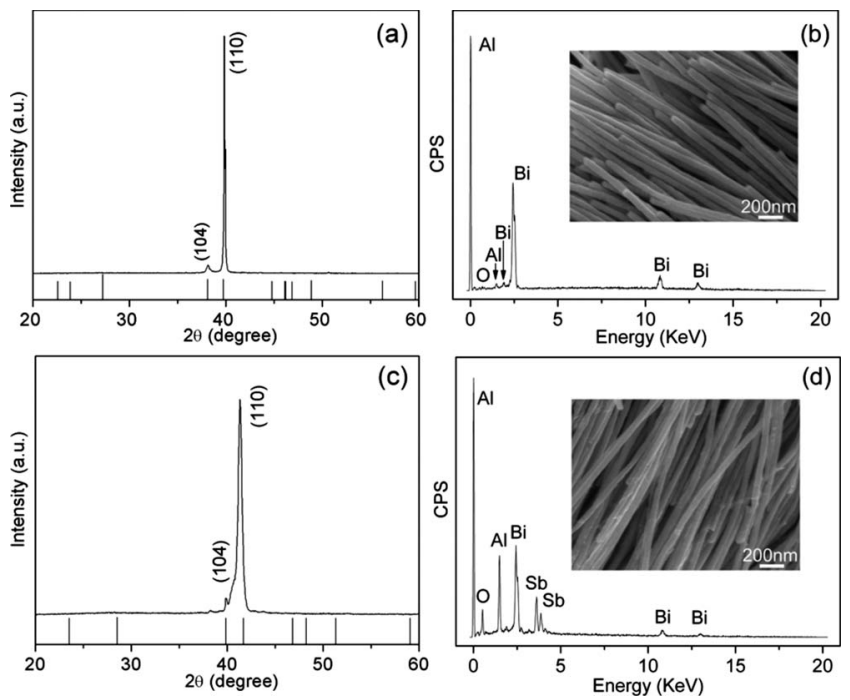

Figure 2. (a) XRD pattern and (b) EDS profile of Bi nanowire array deposited at voltage of $-1.5 \mathrm{~V}$; the inset is the corresponding SEM image. (c) XRD pattern and (d) EDS profile of BiSb nanowire array deposited at a voltage of $-2.0 \mathrm{~V}$; the inset is the corresponding SEM image. 

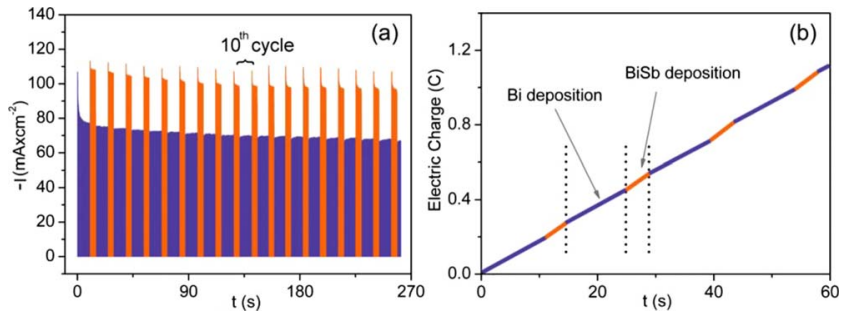

Figure 3. (Color online) Electrochemical characterization of the epitaxial growth of $\mathrm{Bi} / \mathrm{BiSb}$ SLNWs. (a) Typical transient current density as a function of time at modulating voltages of -1.5 and $-2.0 \mathrm{~V}$ and (b) the corresponding passed electric charge as a function of time.

quantitative analysis confirmed that the atomic ratio of $\mathrm{Bi}$ to $\mathrm{Sb}$ in the $\mathrm{Bi}_{1-x} \mathrm{Sb}_{x}$ nanowires is about $1: 1$ with a $5 \%$ error. The FESEM image shows that the diameter of the alloy nanowire is about $60 \mathrm{~nm}$ and is consistent with the AAM pore size.

\section{Results and Discussion}

Electrochemical characterization.- After the optimization of the deposition parameters, modulating voltages of -1.5 and $-2.0 \mathrm{~V}$ were employed to deposit $\mathrm{Bi} / \mathrm{Bi}_{0.5} \mathrm{Sb}_{0.5}$ SLNWs. Figure 3a shows the first 10s of cycles of the cathodic current density as a function of time with the modulating times of $T_{1}=10 \mathrm{~s}$ and $T_{2}=4 \mathrm{~s}$. The higher current density bundle corresponds to the deposition of the $\mathrm{BiSb}$ alloy, while the lower current density bundle corresponds to the deposition of Bi. Each bundle is composed of a series of pulse currents, which correspond to the deposition voltage pulses shown in Fig. 1b. For the first few cycles, the deposition current is relatively high, and the current gradually reaches a relatively stable state and remains nearly constant after about 10 cycles. So, we choose the electric charge passed in the 10th cycle as the voltage switching standard to control the subsequent deposition, upon which to control the bilayer thickness. Note that the deposition time $T_{1}$ or $T_{2}$ constantly changes with the current in the following growth process, indicating an effective control of the RTCAS in maintaining a constant electric charge upon a constant segment length. Figure $3 \mathrm{~b}$ shows the integrated electric charge as a function of time for the deposition of $\mathrm{Bi} / \mathrm{BiSb} \mathrm{SLNWs}$, in which the increased charge and nonlinear change with time can be clearly seen. The line with a smaller slope refers to the deposition of $\mathrm{Bi}$, while that with a larger slope refers to the deposition of BiSb.

The segment lengths of $\mathrm{Bi}$ and $\mathrm{BiSb}$ of $\mathrm{Bi} / \mathrm{BiSb}$ SLNWs can be controlled by appropriately choosing the electric charge according to Faraday's law

$$
L_{\text {actual }}=L_{\text {nominal }} \eta=\left(q M_{\mathrm{a}} / A \rho F n\right) \eta
$$

where $L_{\text {actual }}$ is the actual segment length characterized by TEM, $L_{\text {nominal }}$ is the nominal segment length obtained by Faraday's law $\left(L_{\text {nominal }}=q M_{\mathrm{a}} / A \rho F n\right), q$ is the electric charge passed, $M_{\mathrm{a}}$ is the relative atomic mass, $A$ is the total surface area of the whole array, $\rho$ is the bulk density of the deposited metal, $F$ is Faraday's constant, and $n$ is the number of electrons each ion transferred. The current efficiency $\eta$ for Bi and BiSb segments can be obtained through the above-mentioned optimizing process and is determined by the ratio of the actual arrays' length $L_{\text {actual }}$ measured by FESEM observation and the nominal arrays' length $L_{\text {nominal }}$ obtained by Faraday's law. The $\eta$ for Bi and BiSb segments are about 5-8 and 15-20\%, corresponding to the average growth rates of about 3 and $10 \mathrm{~nm} / \mathrm{s}$, respectively. This result indicates that the current efficiency is relatively low, and the effective reduction in $\mathrm{H}_{2}$ evolution is very important.

EDS composition characterization.-Figure 4 shows the EDS composition profile from left to right along one Bi/BiSb SLNW with a relative large bilayer thickness $\left(L=L_{1}+L_{2}=30+25=55 \mathrm{~nm}\right.$;
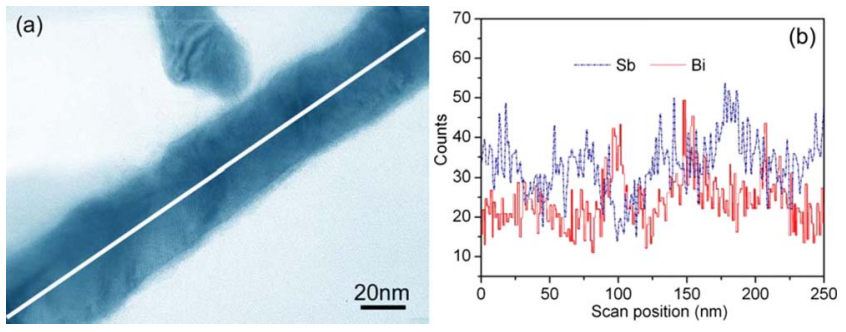

Figure 4. (Color online) (a) TEM image of a Bi/BiSb SLNW with a bilayer thickness of $55 \mathrm{~nm}$ and (b) the corresponding EDS line scan.

modulating times $T_{1}=10 \mathrm{~s}$ and $T_{2}=3 \mathrm{~s}$ ) with the line scanning mode; the periodical change in EDS intensity for $\mathrm{Bi}$ and $\mathrm{Sb}$ from segment to segment with the period of about $55 \mathrm{~nm}$ can be clearly seen. There exist rough layer edges along the SLNW, and this kind of edge was also observed in Ag/Co multilayered nanowires by Valizadeh et al. and was attributed to a combination of different conditions. ${ }^{21}$ Our previous study proved that the diameter of the $\mathrm{BiSb}$ nanowire strongly depends on the deposition voltage; the higher the voltage the smaller the diameter. ${ }^{30}$ From the kinetic perspective of the deposition process, it was considered that at higher deposition voltages, the reduced ions have a shorter diffusing time and, subsequently, a shorter diffusing length on the surface of the nanowire front, which induces a smaller diameter of the BiSb segment, while the lower deposition voltage induces a larger diameter of the Bi segment.

Epitaxial growth characterization.- Figure 5a shows the XRD pattern of the Bi/BiSb SLNW array with the modulating time $T_{1}$ $=T_{2}=1 \mathrm{~s}$. The SLNW array has a [110] preferential orientation, which is consistent with the results of the initial optimization, and further proves that the $\mathrm{Bi}$ and $\mathrm{BiSb}$ segment nanowires grow epitaxially along the $[110]$ direction. ${ }^{38,39}$ Figure $5 \mathrm{~b}$ shows the TEM image of the Bi/BiSb SLNWs with a curved plane growth mode. ${ }^{39}$ The darker regions are the $\mathrm{Bi}$ segments and the brighter ones are the $\mathrm{BiSb}$ segments; the sharp interfaces between $\mathrm{Bi}$ and BiSb layers are clearly seen. Figure 5c shows an HRTEM image of the region marked with a white rectangle in Fig. 5b; the clear and continuous lattice fringes without any dislocations further demonstrate the epitaxial growth of the SLNWs, which also further proves that the wire axis is along the [110] direction. The corresponding SAED pattern shown in Fig. 5d demonstrates that the diffraction points are sharp and bright, indicating the epitaxial growth of the SLNWs. The split-
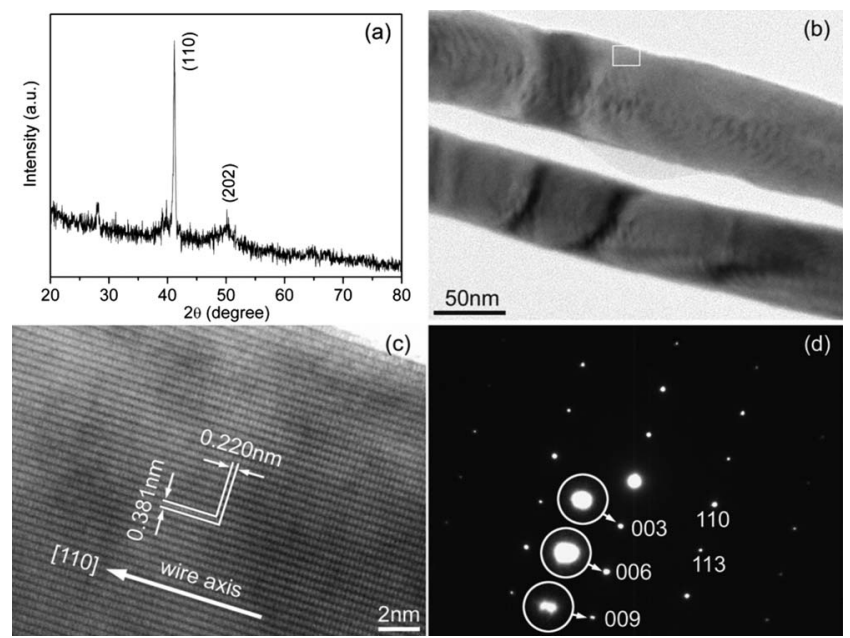

Figure 5. Bi/BiSb SLNWs: (a) XRD pattern, (b) TEM image, (c) HRTEM image, and (d) the corresponding SAED pattern of the area marked in (b). 


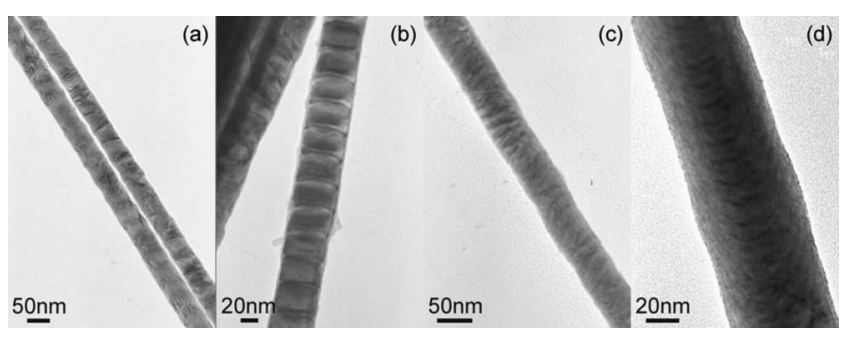

Figure 6. TEM images of the $\mathrm{Bi} / \mathrm{BiSb}$ SLNWs with the modulating times of (a) $T_{1}=5 \mathrm{~s}, T_{2}=2 \mathrm{~s}$; (b) $T_{1}=3 \mathrm{~s}, T_{2}=2 \mathrm{~s}$; (c) $T_{1}=3 \mathrm{~s}, T_{2}=1 \mathrm{~s}$; and (d) $T_{1}=1 \mathrm{~s}, T_{2}=1 \mathrm{~s}$.

ting of the diffraction points along the [003] direction is clearly manifested with the enlarged points, in which the splitting of the superimposition of the diffraction points from the two segments becomes larger with the increase in the lattice index. This result is firm evidence of the layered structure of the SLNWs with a slight difference in the interplanar distance. Similar phenomena have also been observed in the junction region of the GaAs/GaP SLNW ${ }^{13}$ and $\mathrm{InAs} / \mathrm{InP}$ heterostructure ${ }^{14}$ and in epitaxial $\mathrm{Si}-\mathrm{Ge}$ core-shell nanowire heterostructures. ${ }^{40}$ The bilayer thickness of the SLNWs with a curved plane growth mode is about $9 \mathrm{~nm}$ and is smaller than the designed value. The decreased bilayer thickness is attributed to the larger cross-sectional area of the SLNWs with a curved plane growth mode. ${ }^{39}$ It is also evident that the segment length ratio of $\mathrm{Bi}$ to $\mathrm{BiSb}$ is about 1 to 3 .

Controllable bilayer thickness.-Figure 6 shows the typical TEM images of the $\mathrm{Bi} / \mathrm{BiSb}$ SLNWs with different modulating times, in which the bilayer thickness along each SLNW, as well as the smaller bilayer thickness and the sharper segment interface, is more consistent compared to the previous result. ${ }^{24,25}$ The corresponding segment length and the bilayer thickness are listed in Table $\mathrm{I}$, in which the lengths of $\mathrm{Bi}$ and $\mathrm{BiSb}$ segments are nearly equal to the designed ones. This result indicates that the segment length and bilayer thickness of the Bi/BiSb SLNWs can be controlled simply by changing the modulating time, and the consistency of the segment length can be well maintained by our charge-controlled pulse electrodeposition.

Test the Bravais law with SLNWs in the growth of electrodeposited nanowires. - In the final growth stage of the Bi/BiSb SLNWs, spots of gray overgrowth were sometimes observed visually at the template surface, indicating that some wires had reached the upper surface of the AAM template. In this case, the ions would be reduced at these spots preferentially rather than in the front of the nanowires in the AAM pores, which is verified by an obvious difference between the AAM thickness and the SLNW length. Despic et al. ${ }^{41}$ pointed out that the bulge growth easily occurs in an electrodeposition system with a high ion concentration and a high current, and there is an exponential dependence of the growth rate on time.

The growth rate of the electrodeposited nanowires is correlated with the temperature, deposition potential, cathodic current density, and AAM pore size. The nucleation rates of the nanowires in each pore also affect the growth rate of the nanowires because an earlier

Table I. Segment length and bilayer thickness of the Bi/BiSb SLNWs with different modulating times.

\begin{tabular}{lcccc} 
& $T_{1}=5 \mathrm{~s}$, & $T_{1}=3 \mathrm{~s}$, & $T_{1}=3 \mathrm{~s}$, & $T_{1}=1 \mathrm{~s}$, \\
$T_{2}=2 \mathrm{~s}$ & $T_{2}=2 \mathrm{~s}$ & $T_{2}=1 \mathrm{~s}$ & $T_{2}=1 \mathrm{~s}$ \\
\hline$L_{1}(\mathrm{~nm})$ & 17 & 10 & 10 & 3 \\
$L_{2}(\mathrm{~nm})$ & 24 & 20 & 10 & 10 \\
$L(\mathrm{~nm})$ & 41 & 30 & 20 & 13
\end{tabular}

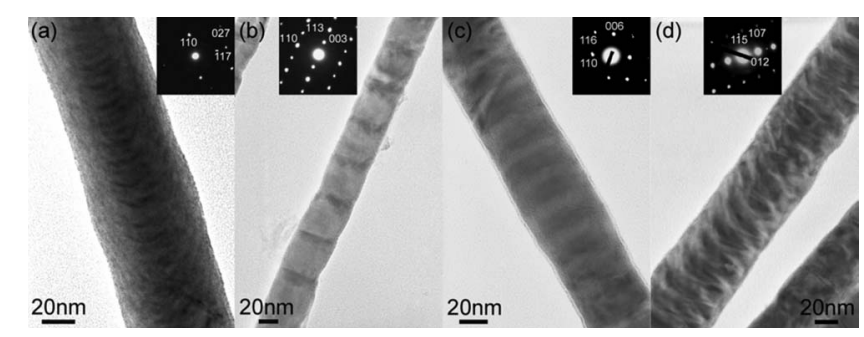

Figure 7. TEM images of the Bi/BiSb SLNWs with different growth orientations and the modulating times of (a) $T_{1}=1 \mathrm{~s}, T_{2}=1 \mathrm{~s}$; (b) $T_{1}=10 \mathrm{~s}$, $T_{2}=6 \mathrm{~s} ;[(\mathrm{c})$ and $(\mathrm{d})] T_{1}=3 \mathrm{~s}, T_{2}=0.5 \mathrm{~s}$.

nucleated nanowire grows preferentially compared to the later ones that have the same orientation. The double growth front observed in some bismuth telluride nanowire arrays was attributed to the different growth rates of the nanowires with different orientations. ${ }^{42}$ The Bravais law defines that the plane with a lower reticular density grows faster than the plane with a higher reticular density under the thermodynamic equilibrium condition. Whether it is still valid under the electrodeposited nanowires needs further proof.

In the XRD patterns of the $\mathrm{Bi} / \mathrm{BiSb} \mathrm{SLNWs}$ one can observe some relative weak diffraction peaks such as (003), (012), (202), (107), and (116), besides the dominant (110) peak. To determine the growth rates of the $\mathrm{Bi} / \mathrm{BiSb} \mathrm{SLNWs}$ with the weak diffraction directions, TEM observations of the $\mathrm{Bi} / \mathrm{BiSb}$ SLNWs were performed on three other samples; the results are shown in Fig. 7. Figure 7a shows the TEM image of the Bi/BiSb SLNW grown along the [110] direction in sample 1 with the modulating time $T_{1}=T_{2}=1 \mathrm{~s}$. Figure $7 \mathrm{~b}$ shows that grown along the [003] direction in sample 2 with the modulating times $T_{1}=10 \mathrm{~s}$ and $T_{2}=6 \mathrm{~s}$. Figure $7 \mathrm{c}$ and $\mathrm{d}$ shows the TEM images of the Bi/BiSb SLNW grown along the [116] and [107] directions, respectively, in sample 3 with the same modulating times $T_{1}=3 \mathrm{~s}$ and $T_{2}=0.5 \mathrm{~s}$. Figure 7 shows that although the SLNWs in these three samples are dominantly along the [110] direction, the Bi/BiSb SLNWs grown along these weak diffraction directions also show an epitaxial growth and can be observed by careful TEM analyses.

Table II summarized the segment length and the growth rate of the $\mathrm{Bi}$ and $\mathrm{BiSb}$ segments of Bi/BiSb SLNWs with different diffraction directions shown in Fig. 7. For the SLNWs with the [110] orientation, the growth rates for segments $\mathrm{Bi}$ and BiSb fit well with the average growth rate shown above. For the SLNWs with a [003] orientation, the growth rate is much lower than the average value. For the SLNWs with a [116] orientation, the growth rate is much higher than the average value, while for those with a [107] orientation, the growth rate is only a little lower than that for SLNWs with a [116] orientation. Because the reticular density from high to low for the planes of (110), (003), (107), and (116) is (003) > (110) $>(107)>(116)$, the above results clearly show that the growth rates of the SLNWs grown along different growth directions obey the Bravais law and demonstrate that the SLNW structure is a model structure to study the growth rates of the electrodeposited nanowires with different growth orientations.

Table II. Segment length and growth rate of the Bi/BiSb SLNWs with different modulating times and growth orientations.

\begin{tabular}{lrrrr} 
& $T_{1}=1 \mathrm{~s}$, & $T_{1}=10 \mathrm{~s}$, & $T_{1}=3 \mathrm{~s}$, & $T_{1}=3 \mathrm{~s}$, \\
& $T_{2}=1 \mathrm{~s}$ & $T_{2}=6 \mathrm{~s}$ & $T_{2}=0.5 \mathrm{~s}$ & $T_{2}=0.5 \mathrm{~s}$ \\
\hline Growth orientation & 110 & 003 & 116 & 107 \\
$L_{1}(\mathrm{~nm})$ & 3 & 11 & 12 & 11.4 \\
$L_{2}(\mathrm{~nm})$ & 10 & 34 & 8 & 6.6 \\
$L_{1} / T_{1}(\mathrm{~nm} / \mathrm{s})$ & 3 & 1.1 & 4 & 3.8 \\
$L_{2} / T_{2}(\mathrm{~nm} / \mathrm{s})$ & 10 & 5.7 & 16 & 13.2
\end{tabular}



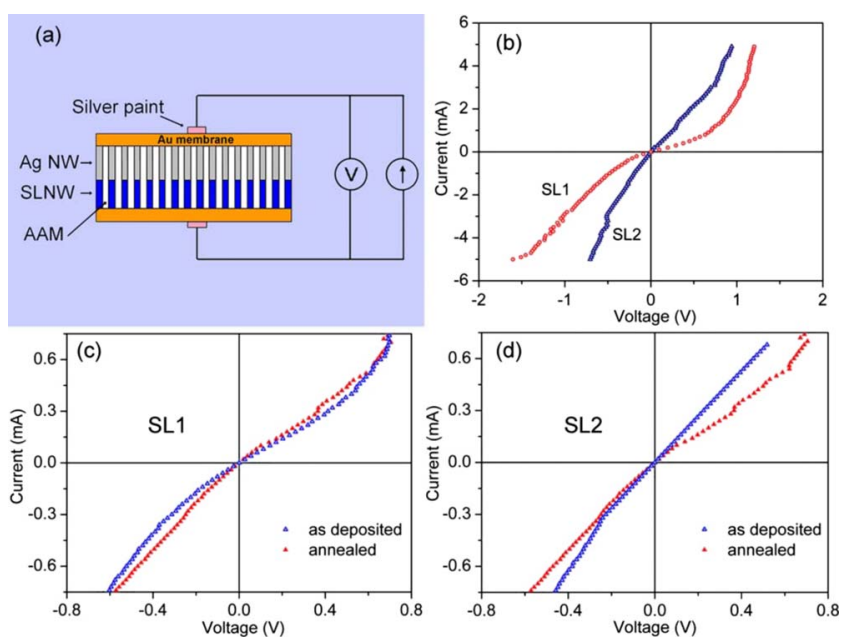

Figure 8. (Color online) (a) Schematic illustration of the $I$ - $V$ curve measurement of the SLNW arrays. (b) $I-V$ curves of samples SL1 and SL2 with a stimulating current range from -5 to $+5 \mathrm{~mA}$. [(c) and (d)] $I-V$ curves of as-deposited and annealed samples SL1 and SL2 with a stimulating current range from -0.75 to $+0.75 \mathrm{~mA}$.

$I$ - $V$ character of SLNWS. - The I-V curves of the SLNW arrays were recorded as a two-point measurement at room temperature, as schematically shown in Fig. 8a. Two typical samples were selected to study the $I-V$ characteristic of the SLNW arrays, one with a large bilayer thickness (the average segment lengths of $\mathrm{Bi}$ and $\mathrm{BiSb}$ are, respectively, 15 and $20 \mathrm{~nm}$, designated as SL1), and the other with a small bilayer thickness (the average segment lengths of $\mathrm{Bi}$ and $\mathrm{BiSb}$ are, respectively, 10 and $5 \mathrm{~nm}$, SL2). To ensure that every nanowire has a good connection, after the growth of the SLNWs (the SLNWs nearly fully filled the pores of the AAM on purpose), the silver nanowires were further deposited by dc plating $\left(\mathrm{AgNO}_{3} 0.25 \mathrm{M}\right.$, $\mathrm{H}_{3} \mathrm{BO}_{3} 0.5 \mathrm{M}, \mathrm{pH} 1.0$, deposition voltage $=-0.5 \mathrm{~V}$ ), and then the gold electrode was plated.

Figure $8 \mathrm{~b}$ shows the typical $I-V$ response of the two samples; one can see that the $I-V$ characteristic is almost symmetrical with respect to the polarity of the applied current. The curve follows a highly nonohmic relation, resembling a metal-semiconductor diode. ${ }^{43}$ This nonlinear increase in voltage with applied current is caused by the Schottky barriers between the SLNWs and the metal electrode in the semiconducting nanowire device and can be explained by the conduction mechanism of the SLNWs. It clearly shows that the obtained $\mathrm{Bi} / \mathrm{BiSb} \mathrm{SLNW}$ are semiconductors, which is favorable to build a thermoelectric device, and also demonstrates that the BiSb alloy segment with an atomic ratio of about 1:1 is proper for thermoelectric application. The slight difference in the $I-V$ response with respect to the polarity of the applied current is considered due to the difference in the Schottky barrier height between gold and silver at the end of the SLNWs. The $I-V$ responses shown in Fig. 8b also demonstrate that the obtained $\mathrm{Bi} / \mathrm{BiSb}$ SLNWs have better electrical conductance compared to a $\mathrm{Bi}$ metal-semiconductor junction nanowire array. ${ }^{44}$ To further analyze the electrical transport properties of the SLNWs, the applied current range was narrowed from -0.75 to $+0.75 \mathrm{~mA}$, and the results are shown in Fig. 8c and d, together with that after the annealing treatment. SL1 exhibits an obvious metal-semiconductor Schottky characteristic in comparison with SL2, and the annealing treatment has almost no influence on the $I-V$ response, indicating that the annealing treatment does not cause a phase separation of the BiSb segment nanowires ${ }^{45}$ and an obvious change in the electrical contacts between the metal and the SLNWs. ${ }^{46}$

The $I-V$ curves shown in Fig. 8 demonstrate the electrical conductance of the Bi/BiSb SLNW array. To further analyze the elec-
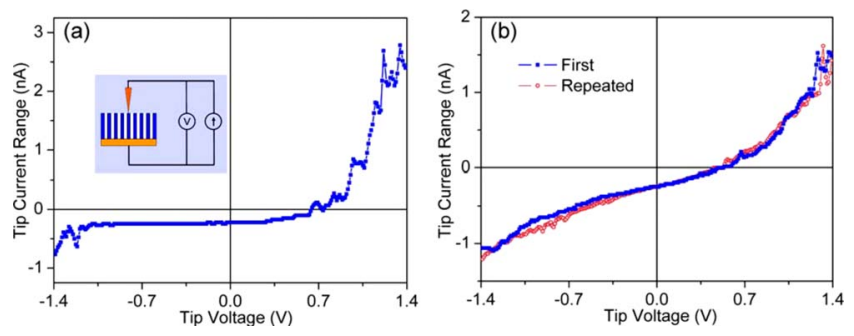

Figure 9. (Color online) $I$ - $V$ response of individual SLNWs embedded in the AAM by STM probe for samples (a) SL1 and (b) SL2. The inset in (a) is the setup for the $I-V$ measurement.

trical conductance of the SLNWs, the $I-V$ response of individual SLNWs embedded in the AAM was characterized by a scanning tunnel microscopy (STM) probe.

Figure 9 shows the typical $I-V$ curves of individual SLNWs in samples SL1 and SL2. The slope of the $I-V$ curve for sample SL1 remains nearly zero at an applied voltage lower than $0.35 \mathrm{~V}$ and increases dramatically at the applied voltage higher than $0.35 \mathrm{~V}$, while that for sample SL2 remains constant at an applied voltage lower than $0.7 \mathrm{~V}$ and increases slowly at applied voltage higher than $0.7 \mathrm{~V}$. The two $I-V$ curves show a typical metal-semiconductor Schottky feature and a marked nonlinearity, and the nonlinearity is more obvious for SL1. This kind of $I-V$ response is very stable and repeatable, as demonstrated in Fig. 9b for the second time measurement. The $I-V$ curves do not pass through the zero point, which might be due to the formation of a thin oxide layer on the surface of the SLNW. This result indicates that the SLNWs with a smaller bilayer thickness have a better electrical conductance and might thus have a better thermoelectric performance.

\section{Conclusions}

Thermoelectric Bi/BiSb SLNWs with a very small bilayer thickness and a sharp segment interface have been grown by adopting the charge-controlled pulse electrodeposition technique. This technique is very effective to ensure the epitaxial growth and the precise design of the SLNWs with a controlling segment length and bilayer thickness, and to maintain the consistency of the segment length. The growth rate of the SLNWs with different growth orientations is different and obeys the Bravais law, which provides important experimental data to the crystal growth theory. The $I-V$ measurement shows that the $\mathrm{Bi} / \mathrm{BiSb} \mathrm{SLNW}$ grown by the present method have a good electrical conductance, particularly that with a smaller bilayer thickness, which is consistent with the theoretical calculation. Our technique will be helpful to those working in the field of precise control of electrodeposited SLNW growth and those pursuing high thermoelectric performance in SLNWs.

\section{Acknowledgment}

This work was supported by the National Natural Science Foundation of China (no. 10474098 and no. 10704074) and the National Major Project of Fundamental Research for Nanomaterials and Nanostructures (no. 2005CB623603).

\section{References}

1. M. S. Dresselhaus, G. Cheng, M. Y. Tang, R. G. Yang, H. Lee, D. Z. Wang, Z. F. Ren, J.-P. Fleurial, and P. Gogna, Adv. Mater. (Weinheim, Ger.), 19, 1043 (2007).

2. L. D. Hicks and M. S. Dresselhaus, Phys. Rev. B, 47, 12727 (1993).

3. R. Venkatasubramanian, E. Siivola, T. Colpitts, and B. O'Quinn, Nature (London), 413, 597 (2001).

4. T. C. Harman, P. J. Taylor, M. P. Walsh, and B. E. LaForge, Science, 297, 2229 (2002).

5. A. Majumdar, Science, 303, 777 (2004)

6. A. I. Boukai, Y. Bunimovich, J. Tahir-Kheli, J.-K. Yu, W. A. Goddard III, and J. R. Heath, Nature (London), 451, 168 (2008).

7. A. I. Hochbaum, R. Chen, R. D. Delgado, W. Liang, E. C. Garnett, M. Najarian, A. Majumdar, and P. Yang, Nature (London), 451, 163 (2008).

8. B. Poudel, Q. Hao, Y. Ma, Y. Lan, A. Minnich, B. Yu, X. Yan, D. Wang, A. Muto, D. Vashaee, et al., Science, 320, 634 (2008). 
9. X. Tang, W. Xie, H. Li, W. Zhao, Q. Zhang, and M. Niino, Appl. Phys. Lett., 90, $012102(2007)$

10. Y.-M. Lin and M. S. Dresselhaus, Phys. Rev. B, 68, 075304 (2003).

11. X. B. Zhao, X. H. Ji, Y. H. Zhang, T. J. Zhu, J. P. Tu, and X. B. Zhang, Appl. Phys. Lett., 86, 062111 (2005).

12. Y. Wu, R. Fan, and P. Yang, Nano Lett., 2, 83 (2002).

13. M. S. Gudiksen, L. J. Lauhon, J. Wang, D. C. Smith, and C. M. Lieber, Nature (London), 415, 617 (2002).

14. M. T. Bjork, B. J. Ohlsson, T. Sass, A. I. Persson, C. Thelander, M. H. Magnusson, K. Deppert, L. R. Wallenberg, and L. Samuelson, Nano Lett., 2, 87 (2002).

15. J. A. Switzer, M. J. Shane, and R. J. Phillips, Science, 247, 444 (1990).

16. L. Piraux, J. M. George, J. F. Despres, C. Leroy, E. Ferain, R. Legras, K. Ounadjela, and A. Fert, Appl. Phys. Lett., 65, 2484 (1994).

17. A. Blondel, J. P. Meier, B. Doudin, and J. P. Ansermet, Appl. Phys. Lett., 65, 3019 (1994).

18. L. Wang, K. Yu-Zhang, A. Metrot, P. Bonhomme, and M. Troyon, Thin Solid Films, 288, 86 (1996)

19. W. Schwarzacher, K. Attenborough, A. Michel, G. Nabiyouni, and J. P. Meier, J. Magn. Magn. Mater, 165, 23 (1997).

20. F. Nasirpouri, P. Southern, M. Ghorbani, A. Iraji zad, and W. Schwarzacher, J. Magn. Magn. Mater, 308, 35 (2007).

21. S. Valizadeh, J. M. George, P. Leisner, and L. Hultman, Thin Solid Films, 402, 262 (2002).

22. S. Valizadeh, L. Hultman, J. M. George, and P. Leisner, Adv. Funct. Mater, 12, 766 (2002).

23. J.-R. Choi, S. J. Oh, H. Ju, and J. Cheon, Nano Lett., 5, 2179 (2005).

24. F. H. Xue, G. T. Fei, B. Wu, P. Cui, and L. D. Zhang, J. Am. Chem. Soc., 127, 15348 (2005).

25. Y. Yoo, X. Feng, N. B. Krassimir, J. Herman, M. A. Ryan, and N. V. Myung, $A d v$. Mater, 19, 296 (2007).

26. J. Cl. Puippe, in Theory and Practice of Pulse Plating, J. Cl. Puippe and F. Leaman, Editors, p. 1, American Electroplaters and Surface Finishers Society, Orlando, FL (1986)
27. C. J. Chen and C. C. Wan, J. Electrochem. Soc., 136, 2850 (1989).

28. Y.-M. Lin, X. Sun, and M. S. Dresselhaus, Phys. Rev. B, 62, 4610 (2000).

29. O. Rabina, Y.-M. Lin, and M. S. Dresselhaus, Appl. Phys. Lett., 79, 81 (2001)

30. X. C. Dou, Y. G. Zhu, X. H. Huang, L. Li, and G. H. Li, J. Phys. Chem. B, 110, 21572 (2006).

31. L. Prieto, M. Martín-González, J. Keyani, R. Gronsky, T. Sands, and A. M. Stacy, J. Am. Chem. Soc., 125, 2388 (2003).

32. M. Martín-González, A. L. Prieto, M. S. Knox, R. Gronsky, T. Sands, and A. M Stacy, Chem. Mater, 15, 1676 (2003).

33. L. Li, G. H. Li, Y. Zhang, Y. W. Yang, and L. D. Zhang, J. Phys. Chem. B, 108, 19380 (2004).

34. F. Gao, Q. Lu, and S. Komarneni, Chem. Commun. (Cambridge), 4, 531 (2005).

35. H. Yu, P. C. Gibbons, and W. E. Buhro, J. Mater. Chem., 14, 595 (2004).

36. K. Nielsch, F. Müller, A.-P. Li, and U. Gösele, Adv. Mater. (Weinheim, Ger.), 12, $582(2000)$.

37. A. Ursache, J. T. Goldbach, T. P. Russell, and M. T. Tuominen, J. Appl. Phys., 97, $10 \mathrm{~J} 322$ (2005).

38. L. Wang, P. Fricoteaux, K. Yu-Zhang, M. Troyon, P. Bonhomme, J. Douglade, and A. Metrot, Thin Solid Films, 261, 160 (1995).

39. X. C. Dou, G. H. Li, and H. C. Lei, Nano Lett., 8, 1286 (2008).

40. L. J. Lauhon, M. S. Gudiksen, D. Wang, and C. M. Lieber, Nature (London), 420, 57 (2002).

41. A. R. Despic, J. W. Diggle, and J. O’M. Bockris, J. Electrochem. Soc., 115, 507 (1968).

42. L. Trahey, C. R. Becker, and A. M. Stacy, Nano Lett., 7, 2535 (2007).

43. Z. Zhang, K. Yao, Y. Liu, C. Jin, X. Liang, Q. Chen, and L. Peng, Adv. Funct Mater, 17, 2478 (2007)

44. L. Li, Y. Zhang, G. H. Li, W. H. Song, and L. D. Zhang, Appl. Phys. A: Mater. Sci. Process., 80, 1053 (2005).

45. Z. Zhang, X. Sun, M. S. Dresselhaus, J. Y. Ying, and J. Heremans, Phys. Rev. B, 61, 4850 (2000).

46. W. Lu and C. M. Lieber, J. Phys. D: Appl. Phys., 39, R387 (2006). 